\title{
ON A CHARACTERIZATION OF TREES
}

\author{
WITOLD D. BULA AND E. D. TYMCHATYN
}

(Communicated by Dennis Burke)

\begin{abstract}
We prove that a continuum $X$ is a tree if and only if for each pair $K \subset L$ of its nondegenerate subcontinua some subcontinuum of $K$ separates $L$.
\end{abstract}

The purpose of this note is to give a slight generalization and a somewhat simpler proof of a recent characterization of trees by Ward [1].

Theorem. A continuum $X$ is a tree if and only if for each pair of nondegenerate subcontinua $K$ and $L$ of $X$ such that $K \subset L$ some subcontinuum of $K$ separates $L$.

A continuum is a compact connected Hausdorff space. A continuum $X$ is a tree if every pair of distinct points of $X$ can be separated by a third point of $X$. A continuum $K$ in a space $X$ is said to be a continuum of convergence of $X$ if there is a net $\left\{K_{\sigma}: \sigma \in \Sigma\right\}$ of subcontinua of $X$ converging to $K$ and such that $K_{\sigma} \cap K=\varnothing$ and either $K_{\sigma}=K_{\tau}$ or $K_{\sigma} \cap K_{\tau}=\varnothing$ for all $\sigma, \tau \in \Sigma$. It is well known and easy to show that a continuum $X$ that contains no continuum of convergence is locally connected.

The continuum $X$ is said to be unicoherent if whenever $X=K \cup L$, where $K$ and $L$ are subcontinua of $X$, then $K \cap L$ is connected. We say that $X$ is hereditarily unicoherent if each of its subcontinua is unicoherent.

Proof of the theorem. Assume that $X$ is a tree. Let $K \subset L$ be nondegenerate subcontinua of $X$ and let $x$ and $y$ be two points of $K$. If $z \in X$ separates $x$ and $y$ then $z \in K$. In particular, $\{z\}$ separates $L$.

Now assume that for each pair $K \subset L$ of nondegenerate subcontinua of $X$ some subcontinuum of $K$ separates $L$. We prove first that $X$ is hereditarily unicoherent. Suppose that $K$ and $L$ are subcontinua of $X$ such that $K \cap$ $L=A \cup B$, where $A$ and $B$ are non-void disjoint closed sets. Let $M$ be a subcontinuum of $K \cup L$ which is irreducible with respect to

(*) both $M \cap K$ and $M \cap L$ are connected and $M \cap A \neq \varnothing \neq M \cap B$.

Received by the editors September 6, 1988 and, in revised form, April 20, 1989.

1980 Mathematics Subject Classification (1985 Revision). Primary 54F20,54F65.

Key words and phrases. Tree, characterization.

This research was supported in part by NSERC grant \#A5616. 
By the Boundary Bumping Theorem (see $[2, \mathrm{p} .16]$ ) there is a nondegenerate continuum $N \subset M \backslash L$. Let $P$ be a subcontinuum of $N$ and suppose that $M \backslash P=U \cup V$, where $U$ and $V$ are non-empty disjoint open sets in $M$. The set $M \cap L$ is connected, so we may suppose $M \cap L \subset U$. Hence, $(A \cup B) \cap M \subset U$ and $V \subset K \backslash L$. Also, $(M \cap K) \backslash P=(M \cap K \cap U) \cup(M \cap K \cap V)$, the union of two separated sets, so $(M \cap K \cap U) \cup P=(U \cup P) \cap K$ is connected. Hence, $U \cup P$ is a proper subcontinuum of $M$ satisfying $(*)$. This is a contradiction. Thus, $N$ is a nondegenerate subcontinuum of $M$, no subcontinuum of which separates $M$ contrary to the hypothesis of the theorem. Thus, every pair of subcontinua of $X$ intersects in a connected set. It follows that for every pair $(x, y)$ of distinct points of $X, x$ and $y$ are joined by a unique smallest continuum.

Suppose $X$ contains a nondegenerate continuum of convergence $K$. Let $\left\{K_{\sigma}: \sigma \in \Sigma\right\}$ be a net of subcontinua in $X$ converging to $K$ and such that $K_{\alpha} \cap K=\varnothing$ and either $K_{\alpha} \cap K_{\beta}=\varnothing$ or $K_{\alpha}=K_{\beta}$ for $\alpha, \beta \in \Sigma$. For each $\sigma \in \Sigma$ let $M_{\sigma}$ be a subcontinuum of $X$ which irreducibly joins $K$ and $K_{\sigma}$. By the above $M_{\sigma} \cap K$ is connected. By the irreducibility of $M_{\sigma}$ no subcontinuum of $M_{\sigma} \cap K$ separates $M_{\sigma}$. By the hypothesis $M_{\sigma} \cap K=\left\{p_{\sigma}\right\}$ for some $p_{\sigma} \in K$. Let $\mathscr{U}$ be a finite cover of $K$ by open sets in $K$ whose closures do not contain $K$. For $U \in \mathscr{U}$ let $\Sigma(U)=\left\{\sigma \in \Sigma: p_{\sigma} \in U\right\}$. Since $\mathscr{U}$ is finite $\Sigma(W)$ is cofinal in $\Sigma$ for some $W \in \mathscr{U}$. Without loss of generality we may assume $\Sigma(W)=\Sigma$. Let $C$ be a nondegenerate continuum in $K \backslash \mathrm{cl} W$. Let $L=\operatorname{cl}\left(\bigcup\left\{K_{\sigma} \cup M_{\sigma}: \sigma \in \Sigma\right\}\right)$. It is easy to check that $C$ contains no separating subcontinuum of $L$ contrary to the hypothesis of the theorem. Hence, $X$ is locally connected.

Finally we prove that $X$ is a tree. Let $x$ and $y$ be two points of $X$. Let $K$ be the smallest continuum in $X$ from $x$ to $y$. Let $z \in K \backslash\{x, y\}$. If $z$ did not separate $x$ from $y$ in $X$ it would follow from the local connectedness and the regularity of $X$ and the Chaining Lemma (see [2, p.13]) that there is a continuum $L \subset X \backslash\{z\}$ such that $x, y \in L$. This would contradict the hereditary unicoherence of $X$. Hence, the point $z$ separates $x$ from $y$ in $X$.

Corollary (Ward). A continuum $X$ is a tree if and only if for each pair of nondegenerate subcontinua $K$ and $L$ of $X$ such that $K \subset L$ some point of $K$ separates $L$.

\section{REFERENCES}

1. L.E.Ward, Jr., A new characterization of trees, Proc. Amer. Math. Soc. 104 (1988), 12521255 (to appear).

2. G.T.Whyburn, Analytic topology, Amer. Math. Soc. Colloq. Publ., New York, 1942.

Department of Mathematics, University of Saskatchewan, Saskatoon, Saskatchewan S7N OW0 CANADA 